Annuaire suisse de politique de développement

23-2 | 2004

Les ONG de développement : rôles et perspectives

\title{
Le rôle des ONG dans la politique de développement de la Suisse
}

Joseph Deiss et Walter Fust

\section{(2) OpenEdition}

1 Journals

Édition électronique

URL : http://journals.openedition.org/aspd/457

DOI : 10.4000/aspd.457

ISSN : 1663-9669

Éditeur

Institut de hautes études internationales et du développement

Édition imprimée

Date de publication : 1 novembre 2004

Pagination : 117-125

ISSN : 1660-5934

Référence électronique

Joseph Deiss et Walter Fust, « Le rôle des ONG dans la politique de développement de la Suisse »,

Annuaire suisse de politique de développement [En ligne], 23-2 | 2004, mis en ligne le 10 mars 2010

consulté le 08 septembre 2020. URL : http://journals.openedition.org/aspd/457 ; DOI : https://doi.org/ $10.4000 /$ aspd. 457 


\title{
Le rôle des ONG
}

\section{dans la politique de développement de la Suisse ${ }^{1}$}

\author{
Joseph Deiss* et Walter Fust**
}

\section{Introduction}

L'évolution de la société, l'intensification des relations internationales et la mondialisation confèrent une importance croissante aux organisations de la société civile. Les acteurs non étatiques, en particulier les organisations non gouvernementales (ONG), jouent ainsi un rôle de plus en plus influent dans la politique étrangère. Et cette remarque vaut aussi pour la coopération au développement et l'aide humanitaire. Or, c'est justement dans ces domaines que les organisations privées suisses - que ce soit par leurs propres activités ou par leur collaboration avec les organismes de l'Etat - relèvent d'une tradition qui remonte à des décennies. Les ONG attirent l'attention sur des sujets et des préoccupations qui n'ont pas une place centrale dans l'actualité politique car ils ne peuvent compter que sur leur propre groupe de pression. Cet état de fait a motivé la fondation de nombreuses auvres d'entraide privées et constitue aujourd'hui encore l'un des points forts des organismes de développement.

\section{Les origines de la coopération suisse au développement}

«Considérons l'aide au développement de la Suisse dans son ensemble. Nous constatons avec joie qu'aussi bien les activités de l'Etat que celles des particuliers vont aujourd'hui de soi. Cette aide au développement constitue par ailleurs une part essentielle de la politique extérieure de la Suisse.»

C'est en ces termes que Regina Käki-Fuchsmann, une pionnière de la coopération privée suisse au développement, entamait le bilan qu'elle dressait en 1964. Pendant des années (de 1934 à 1951), Regina Kägi-Fuchsmann a dirigé l'Euvre suisse d'entraide ouvrière. Elle a appartenu au cercle de personnes qui se sont dépensées sans compter dans les années 1950 - persuadées qu'elles étaient que l'aide au développement devait compter parmi les tâches nationales de la Suisse - pour créer un organisme non gouvernemental dans ce domaine. Leurs efforts ont abouti à la fondation, en 1955, d'une association confessionnellement et politiquement neutre: l'Aide suisse aux régions extra-européennes (Helvetas dès 1965).

1 Cet article a été publié pour la première fois en 2003 sous le titre original «Zur Rolle der NGOs in der Entwicklungspolitik der Schweiz», dans l'ouvrage Öffentliche Verwaltung und Nonprofit-Organisationen, hommage à Reinbert Schauer, édité par Ernst-Bernd Blümle, Helmut Pernsteiner, Robert Purtschert, René Clemens Andessner, Linde Verlag, Vienne, 2003, pp. 103-114. Nous remercions la maison Linde Verlag pour son aimable autorisation de reproduire cet article.

* Conseiller fédéral en charge du Département fédéral de l'économie.

** Directeur de la Direction du développement et de la coopération (DDC). 
Voici les arguments avancés par les promoteurs de l'Aide suisse aux régions extra-européennes pour motiver leur initiative:

- l'étendue et le type de l'aide fournie aux pays économiquement sous-développés revêtent une importance décisive pour l'avenir du monde entier;

๖ il importe d'amener le peuple suisse à prendre conscience de la situation mondiale, en particulier des liens étroits qui unissent tous les continents dans les domaines économiques, sociaux et culturels, et d'inviter le peuple suisse à fournir une aide solidaire aux pays qui en ont besoin.

La coopération privée suisse au développement a également vu le jour dans les années 1950: diverses œuvres d'entraide suisses ont été créées pour venir en aide aux régions d'Europe détruites par la guerre. Caritas, l'Entraide protestante suisse (EPER), l'Euvre suisse d'entraide ouvrière (OSEO), la Croix-Rouge suisse et Swissaid ont été les principales d'entre elles. Dans les années 1960, ces organisations sont ensuite devenues des acteurs de poids dans l'aide privée au développement.

L'économie privée a aussi apporté sa pierre à l'édifice de l'aide non gouvernementale, puisqu'elle finance la Fondation suisse d'assistance au développement technique, qui a été fondée en 1959 (nommée Swisscontact par la suite).

Parmi les précurseurs de l'aide privée au développement, il convient enfin de mentionner les missionnaires suisses.

Le fait que des organisations privées sans but lucratif aient choisi de promouvoir et de soutenir la coopération suisse au développement ne découle pas seulement de la tradition humanitaire de la Suisse. Il s'agit bien plus des effets d'une prise de conscience face à la situation économique et sociale dans les pays du Sud, en particulier au sein des nations qui s'émancipaient peu à peu de la tutelle coloniale. On a alors compris pour la première fois que la situation socio-économique de ces pays constituait non seulement un problème d'ampleur internationale mais aussi une menace pour la paix dans le monde.

\section{L'ancrage des ONG dans la société}

Les organismes privés de développement se distinguent surtout par leur profond ancrage dans la société suisse, qui repose notamment sur les quatre piliers suivants :

- la capacité à mobiliser des membres et des groupes locaux (Aide suisse aux régions extra-européennes, aujourd'hui Helvetas);

- les activités de milieux religieux, issus de l'aide aux pays européens (EPER, Caritas) ou de mouvements laïcs catholiques (Action de Carême);

- l'engagement d'organismes ouvriers, issus de la tradition des secours aux ouvriers et aux enfants en Europe (OSEO);

๖ la participation de l'économie privée (Swisscontact).

Par la diversité de leur assise sociale, les organisations non gouvernementales (ONG) offrent une base nationale solide à la coopération suisse au développement.

Si le gouvernement suisse (le Conseil fédéral) a d'emblée témoigné sa bienveillance et son appui à l'égard des promoteurs de l'aide privée au développe- 
ment, les efforts déployés par les organisations privées ont largement favorisé l'engagement croissant de l'Etat dans ce domaine. C'est ainsi qu'a été créé, en 1961, le Service de coopération technique au sein du Ministère des affaires étrangères, le Département politique (devenu aujourd'hui Département fédéral des affaires étrangères, DFAE). Au fil des décennies, ce service s'est transformé pour devenir l'actuelle Direction du développement et de la coopération (DDC). La même année, le Parlement a accordé un premier crédit de 60 millions de francs destiné à l'aide au développement, mettant ainsi en place les bases organisationnelles et matérielles d'une étroite collaboration et d'un véritable partenariat entre acteurs publics et privés de la coopération au développement.

Le rôle des ONG figure expressément dans la Loi fédérale sur la coopération au développement et l'aide humanitaire internationales du 19 mars 1976. Dans les différents messages qu'il a adressés au Parlement concernant la poursuite de la coopération technique et de l'aide financière en faveur des pays en développement, le Conseil fédéral n'a d'ailleurs pas manqué de souligner l'importance cruciale du partenariat avec les ONG, tant dans le dialogue politique et dans les échanges d'expériences que dans la mise en œuvre de la coopération suisse au développement.

\section{Caractéristiques et spécificités des ONG suisses}

Les ONG suisses à l'œuvre dans la coopération au développement comprennent environ 30 organisations relativement grandes et 150 plus petites, qui se distinguent par leur orientation thématique et conceptuelle. L'éventail va des œuvres d'entraide opérant de manière professionnelle aux mouvements solidaires dont l'action repose essentiellement sur le bénévolat. Certaines de ces organisations possèdent un rayonnement national, tandis que d'autres s'appuient avant tout sur des bases régionales, voire locales. Le rayon d'action varie selon l'organisation: il peut comprendre le monde entier ou, au contraire, être géographiquement très limité. Malgré cette grande variété, Il est possible d'esquisser un paysage des ONG suisses actives dans la coopération au développement.

Commençons cependant par relever les principales caractéristiques d'une ONG suisse. Qu'est-ce qui distingue une ONG d'une entreprise privée ou d'une société-conseil? En premier lieu, le fait qu'une ONG n'a pas de but lucratif et possède donc une structure organisationnelle différente. Les ONG ont par ailleurs pour objectif d'induire un changement dans la société. Elles visent ainsi à exercer une influence sur les structures économiques et sociales du pays.

Elles se considèrent comme des acteurs à part entière des processus politiques et sociaux. Et c'est là aussi que réside la différence, en matière de politique de développement, entre les places respectives qu'occupent une ONG et une entreprise privée.

On peut classer les œuvres d'entraide et les organisations privées suisses actives dans la coopération au développement dans les grandes catégories suivantes:

1. Organisations axées sur la politique de développement: Communauté de travail des auvres d'entraide et Déclaration de Berne.

Ces organisations s'attachent avant tout à analyser la politique de développement, à assurer un travail d'information et à promouvoir une certaine prise de 
conscience au sein de la population. Elles entretiennent le dialogue avec l'Etat et l'économie privée et contribuent au débat sur la politique de développement sur les plans national et international.

2. Organismes voués aux activités opérationnelles de la coopération au développement: Helvetas, Swissaid, Swisscontact, Intercooperation.

Ces organismes réalisent de vastes programmes de développement en Afrique, en Asie et en Amérique latine. Ils assurent aussi, à des degrés divers, un travail d'information et de sensibilisation en Suisse.

3. Euvres d'entraide multifonctionnelles possédant un département consacré à la coopération au développement et à l'aide humanitaire: elles comprennent les œuvres d'entraide religieuses - dont Caritas et EPER - ainsi que des organismes non confessionnels tels que la Croix-Rouge suisse et l'Euvre suisse d'entraide ouvrière.

Outre leurs activités à l'étranger, ces organismes effectuent aussi un travail social considérable en Suisse. Ils assurent également un travail d'information et de sensibilisation en Suisse.

4. Organisations spécialisées dans l'aide aux enfants, comme Terre des Hommes. Ces organisations fournissent de l'aide humanitaire et réalisent des programmes de développement en Asie, en Afrique et en Amérique latine. Leur travail se fonde essentiellement sur la Convention sur les droits de l'enfant de l'ONU.

5. Organisations écologistes et de protection de l'environnement, telles que le WWF Suisse ou Pro Natura. Ces organisations défendent un développement respectueux de l'environnement et la protection de la nature en Suisse et à l'étranger.

6. Organisations fondées sur le bénévolat et d'orientations diverses, réunies au sein de la plate-forme Unité. Ces organisations misent sur les échanges de personnes dans les activités les plus variées allant de programmes de soutien techniques et professionnels à la promotion des échanges et du dialogue interculturels.

La Suisse compte par ailleurs un grand nombre d'organismes locaux et de groupes d'entraide plus petits. Ceux-ci mènent les activités les plus variées sur le plan international dans les domaines humanitaire, social et du développement. Des fédérations cantonales d'ONG ont aussi vu le jour dans certains cantons, tels Genève, Vaud, le Jura et le Tessin. Ces fédérations favorisent le travail opérationnel avec leurs membres et le dialogue sur la politique de développement avec les autorités locales.

Toutes ces organisations partagent un même but: promouvoir un développement durable, lutter contre l'injustice et contre la pauvreté et atténuer les inégalités sociales.

Le volume des dons que les $\mathrm{ONG}$ suisses parviennent à mobiliser atteint quelque 250 millions de francs par an. Sur ce total, 190 millions sont consacrés à la coopération au développement et 60 millions à l'aide humanitaire. 


\section{Collaboration sur le terrain}

La DDC soutient les programmes de développement des 13 plus grandes ONG suisses et organisations faîtières d'ONG en leur versant des contributions sur plusieurs années. Les ONG formulent elles-mêmes les objectifs de leurs programmes, définissent leur déroulement, choisissent leurs partenaires dans les pays en développement et garantissent la qualité de leurs programmes. Les contributions de la DDC sont liées à des prestations financières fournies par les organisations elles-mêmes.

Ces contributions ne sont pas des subventions. Il s'agit de montants négociés destinés à des programmes dotés d'objectifs clairement définis et ils sont en fait versés sur la base de véritables contrats de prestations.

Pour la période 2002 à 2004, les contributions de la DDC à des programmes d'ONG atteindront en moyenne 60 millions de francs par an, soit l'équivalent de $14 \%$ du budget de la DDC pour la coopération bilatérale au développement.

Les ONG plus petites ayant fait leurs preuves peuvent obtenir des contributions destinées à des projets spécifiques. Les dépenses annuelles de la DDC consacrées à ce type de soutien se situent entre 3 et 4 millions de francs.

La Confédération charge parfois aussi les ONG de réaliser ses projets de développement. Dans ce cas, les ONG concluent un contrat avec la Confédération. Les principaux partenaires contractuels de la DDC sont Helvetas, Intercooperation et Swisscontact. En 2000, les mandats de la DDC confiés à des ONG suisses ont atteint un volume global de 71 millions de francs. Parmi les autres mandataires de la DDC, on trouve des universités, des instituts spécialisés et des sociétés-conseils privées.

Dans la seconde moitié des années 1990, les ONG suisses ont mis en œuvre environ $25 \%$ de la coopération bilatérale de la DDC.

\section{Echange d'expériences et dialogue politique}

ONG et DDC entretiennent un dialogue permanent et échangent régulièrement leurs expériences. Ces interactions, sur des thèmes précis présentant un intérêt commun, favorisent la complémentarité et les synergies et remettent sans cesse en question l'adéquation des stratégies et des programmes.

Grâce à leur expérience du terrain et à leurs études de fond, les ONG jouent un rôle crucial pour alimenter le débat politique sur le développement et contribuent à améliorer la qualité de la coopération suisse au développement. Leurs critiques constructives sur des points essentiels revêtent dans ce cadre une importance toute particulière.

On peut illustrer l'importance politique des ONG en rappelant par exemple le soutien qu'elles ont apporté à l'adhésion de la Suisse aux Institutions de Bretton Woods, à l'OMC et à l'ONU. Lors de la campagne en faveur de l'adhésion de la Suisse à l'ONU, la Communauté de travail des œuvres d'entraide a ainsi soutenu l'initiative populaire allant dans ce sens et largement contribué à réunir les signatures nécessaires à sa validation. Elle a également pris une part active à la 
campagne qui a précédé le vote par lequel le peuple suisse s'est prononcé le 3 mars 2002 en faveur de l'adhésion à l'ONU.

Les critiques et les divergences d'opinion font bien entendu aussi partie du dialogue politique. Les sujets controversés comprennent en particulier les positions défendues par la Suisse au sein de l'OMC, les relations commerciales en général, le droit des brevets, les exportations de matériel de guerre, la Garantie contre les risques à l'exportation et l'entraide judiciaire internationale.

\section{L'indépendance des ONG}

L'indépendance des ONG repose avant tout sur leur autonomie financière. Les contributions et les mandats des pouvoirs publics (Confédération, cantons et communes) occupent cependant une grande place dans leurs recettes. $\mathrm{Vu}$ la variété et la multifonctionnalité des ONG suisses, il est toutefois impossible de dire dans quelle mesure elles dépendent des fonds publics.

En matière de coopération au développement, la Confédération accorde une grande importance aux prestations financières des ONG. Elle ne veut pas, en effet, que ses contributions puissent être utilisées pour garantir le maintien des ONG en Suisse, car l'argent est destiné en priorité à servir les objectifs de la politique de développement et des programmes financés.

L'indépendance des ONG comprend aussi la libre expression de leur opinion dans le dialogue politique, une liberté qui relève de leur nature même. Des tensions peuvent toutefois apparaître lorsque le dialogue porte sur des sujets politiquement sensibles ou lors de campagnes précédant des votations. L'indépendance des ONG ne repose donc pas uniquement sur les finances, mais dépend aussi de la marge de manœuvre que l'on réserve en démocratie à des opinions politiques divergentes. La pluralité de la société suisse doit en effet pouvoir se refléter dans la politique de développement du pays. Force nous est de reconnaître que si nous voulons vraiment promouvoir les droits de l'homme, la démocratie et la bonne gestion gouvernementale tout en renforçant les structures de la société civile, nos efforts ne seront crédibles que si nous prenons au sérieux les organisations de notre propre société civile, non seulement dans leur rôle de critiques, de pionnières, voire de partenaires coriaces lors de la prise de décisions politiques, mais aussi lorsqu'elles défendent les intérêts de la population.

Leur participation éclairée au dialogue politique fait donc également partie de leur indépendance.

\section{Les ONG : pionnières dans leur domaine et moteur de la société civile}

Les organisations non gouvernementales sont désormais des intervenants de premier plan sur la scène internationale. Elles ont tissé des réseaux internationaux toujours plus denses, elles deviennent peu à peu des partenaires non négligeables dans le dialogue avec les organisations multilatérales et leur avis acquiert de plus en plus de poids pour ce qui est de la gouvernance mondiale et des réglementations internationales.

Les ONG ont souvent joué un rôle de pionnières et représenté la volonté de la société civile. Voici quelques exemples marquants pour illustrer notre propos: 
- Handicap International a compté parmi les principaux acteurs de la campagne internationale contre les mines. Cette campagne est aujourd'hui financée par 1300 organisations de plus de 90 pays et sa coordination est assurée par un comité composé de plusieurs ONG. Leurs efforts communs ont conduit à l'adoption, en 1997, de la Convention sur l'interdiction de l'emploi, du stockage, de la production et du transfert des mines antipersonnel. Sur les 142 Etats signataires de la convention, 122 l'ont ratifiée jusqu'en octobre 2001 (dont la Suisse en 1998). La campagne a aussi donné naissance à un autre réseau d'information fondé sur la société civile. Ce réseau permet de suivre l'application de la convention de même que l'évolution de la situation pour ce qui est des mines en général. Dans ce domaine, les ONG sont parvenues à susciter une dynamique que les gouvernements n'auraient pu générer à eux seuls. La Suisse apporte son soutien au programme mené par Handicap International en Asie du Sud-Est pour venir en aide aux victimes des mines, un programme également issu de la campagne mondiale.

๖ Dans les années 1980, les ONG suisses ont lancé une campagne pour le désendettement. Menée par 26 organisations suisses, cette campagne demandait au Conseil fédéral d'annuler toutes les dettes des pays pauvres envers la Suisse. Une pétition, intitulée «Le désendettement: une question de survie», a réuni 250'000 signatures. Elle a poussé le Parlement à compléter le créditcadre destiné à la coopération au développement par un véritable programme de désendettement qui a été adopté en 1991, à l'occasion du 700e anniversaire de la Confédération. Grâce à l'initiative des ONG, les programmes de désendettement font désormais partie intégrante de la coopération suisse au développement. La participation active de la Suisse au débat international sur le désendettement a d'ailleurs contribué à inciter la Banque mondiale à revoir sa politique et à prendre des mesures en faveur du désendettement. Dans ce domaine, les ONG étaient donc en première ligne et le rayonnement de leurs efforts dépasse aujourd'hui largement le cadre de la coopération suisse au développement.

Ces deux exemples montrent que la pression de la société civile et d'âpres discussions politiques débouchent souvent sur des réalisations historiques.

A une époque où les problèmes mondiaux - notamment ceux liés à l'environnement et aux migrations - influent grandement sur la politique nationale, la politique de développement ne peut se passer d'un débat franc ni des désaccords qui l'accompagnent. Elle a besoin du partenariat avec la société civile et avec le secteur privé, car elle doit pouvoir se fonder sur un dialogue critique et constructif entre l'Etat, la société civile et l'économie privée. Il lui faut aussi une discussion ouverte sur les problèmes de développement et sur les corrélations structurelles, une discussion qui serve à rechercher ensemble les solutions qui permettront de relever les défis qui nous attendent.

Des ONG actives sur le terrain et dans le domaine des idées contribuent grandement à trouver de nouvelles approches et à ouvrir de nouvelles voies en matière de coopération. Il importe dès lors de veiller en priorité à ce que la confrontation issue des critiques adressées à la mondialisation - de Seattle à Gênes en passant par Davos - ne conduise pas à une polarisation et à un renforcement des fronts entre Etat et ONG. Cette confrontation doit au contraire servir de fondement à un débat ouvert sur ses origines profondes. Les événements évoqués 
soulignent aussi la nécessité d'élargir la plate-forme du dialogue, de renforcer des alliances et d'intégrer les organisations de la société civile - en tant que partenaires critiques, voire coriaces - à une collaboration basée sur un véritable partenariat.

\section{Perspectives}

L'évolution rapide du contexte mondial place la coopération au développement face à de nouvelles difficultés. De nouveaux fossés sociaux apparaissent alors que des systèmes de référence socioculturels s'érodent. L'élargissement des marges de manœuvre à l'échelle mondiale provoque un sentiment d'impuissance au niveau social, ouvrant non seulement la voie à de nouvelles formes de protestation et d'opposition, mais augmentant aussi les risques d'une dérive extrémiste. Par ailleurs, la lutte pour les biens publics mondiaux, telle l'eau, s'accentue. Enfin, l'Etat, la société civile et l'économie privée se doivent de relever un défi de taille: assumer ensemble la durabilité du développement à venir.

L'économie et le commerce doivent être dotés de structures qui permettent d'éviter la pauvreté. Un environnement sain et l'exploitation réfléchie des ressources naturelles constituent les fondements d'un développement durable et ne doivent en aucun cas être subordonnés à des intérêts économiques. L'équité sociale et l'Etat de droit doivent servir à surmonter les oppositions sociales et régionales et à prévenir la violence. Dans ce contexte, les ONG sont en mesure de favoriser grandement les changements politiques et économiques qui s'imposent et de les promouvoir aux niveaux tant local que régional, voire mondial.

Si les autorités suisses tiennent à promouvoir un dialogue critique et politique avec les ONG sur la politique de développement et à collaborer avec elles sur le terrain, elles ont cependant aussi des exigences à l'égard des ONG. Voici ce que celles-ci doivent faire pour les satisfaire:

口 se distancer de la violence dans le cadre des confrontations politiques;

$\checkmark$ être capables de mener des analyses sérieuses et de mettre en évidence des solutions applicables;

$\checkmark$ posséder des structures claires et baser leur gestion et leur comptabilité sur la transparence;

- évaluer leur travail sur la base de leur capacité à réaliser les objectifs et se doter d'un système efficace de gestion de la qualité.

Les ONG doivent rester des ONG. Nous ne voulons pas d'ONGOG (organisations non gouvernementales organisées par le gouvernement), nous voulons des partenaires issus de la société civile qui soient dignes de ce nom. 
Tableau 1: Aide publique et privée de la Suisse au développement, 1995-2001 (en millions de francs suisses)

\begin{tabular}{|c|c|c|c|c|c|c|c|}
\hline & 1995 & 1996 & 1997 & 1998 & 1999 & 2000 & $2001^{p}$ \\
\hline Aide privée au développement & 219.3 & 226.6 & 225.7 & 250.0 & 275.4 & 285.4 & n.c. ${ }^{*}$ \\
\hline \multicolumn{8}{|l|}{ Aide publique au développement } \\
\hline Confédération & 1261.4 & 1250.5 & 1298.0 & 1279.9 & 1444.7 & 1479.9 & $1509.0^{p}$ \\
\hline aide bilatérale de la DDC & 488.6 & 479.4 & 489.1 & 488.1 & 465.6 & 472.8 & 502.0 \\
\hline contributions et mandats de la & 124.4 & 112.3 & 132.1 & 119.1 & 125.1 & 127.5 & 128.1 \\
\hline DDC à des ONG (aide bilatérale) & $25.5 \%$ & $23.4 \%$ & $26.9 \%$ & $24.4 \%$ & $26.8 \%$ & $27.0 \%$ & $25.5 \%$ \\
\hline Cantons & 11.2 & 9.6 & 15.1 & 13.2 & 11.6 & 14.1 & 13.6 \\
\hline Communes & 8.2 & 8.6 & 7.8 & 8.4 & 8.9 & 8.1 & 7.5 \\
\hline Total & 1280.8 & 1268.7 & 1320.9 & 1301.5 & 1465.2 & 1502.1 & $1530^{p}$ \\
\hline $\begin{array}{l}\text { Aide privée au développement (en \% } \\
\text { de l'aide publique au développement }\end{array}$ & $\begin{array}{l}0 \\
\text { t) } 17.1\end{array}$ & 17.9 & 17.1 & 19.2 & 18.8 & 19.0 & n.c. ${ }^{*}$ \\
\hline $\begin{array}{l}\text { Aide privée au développement } \\
\text { (en \% du produit national brut) }\end{array}$ & 0.06 & 0.06 & 0.06 & 0.06 & 0.07 & 0.07 & n.c. ${ }^{*}$ \\
\hline $\begin{array}{l}\text { Aide publique au développement } \\
\text { (en \% du produit national brut) }\end{array}$ & 0.34 & 0.34 & 0.34 & 0.32 & 0.35 & 0.34 & 0.34 \\
\hline
\end{tabular}

Remarque: Les montants de l'aide privée au développement représentent les moyens financiers fournis par les œuvres d'entraide sans les contributions publiques.

${ }^{p}$ montant provisoire.

* n.c. : montant non encore connu.

Source: DDC, avril 2002. 